\title{
Efeito alelopático de folhas de Solanum lycocarpum A. St.-Hil. (Solanaceae) na germinação e crescimento de Sesamum indicum $\mathbf{L}$. (Pedaliaceae) sob diferentes temperaturas
}

\author{
Sarah C. Caldas Oliveira ${ }^{1}$, Alfredo Gui Ferreira ${ }^{2}$ e Fabian Borghetti ${ }^{3}$
}

Recebido em 15/02/2003. Aceito em 01/11/2003

\begin{abstract}
RESUMO - (Efeito alelopático de folhas de Solanum lycocarpum A. St.-Hil. (Solanaceae) na germinação e crescimento de Sesamum indicum L. (Pedaliaceae) sob diferentes temperaturas). Alelopatia pode ser definida como o efeito maléfico ou benéfico que uma planta exerce sobre a outra por meio de compostos químicos liberados no ambiente. Diversas espécies do gênero Solanum apresentam evidências de propriedades alelopáticas. S. lycocarpum A. St.-Hil (lobeira) é espécie de ampla distribuição em ambientes perturbados do Cerrado. No presente trabalho foram investigados efeitos alelopáticos de extratos de folhas de lobeira na germinação e no crescimento do gergelim (Sesamum indicum L.). Extratos aquosos das folhas foram preparados nas concentrações de 1\%, 2\%, 3\%, 4\% e 5\% (p/v). A osmolaridade dos extratos foi medida e soluções de polietileno glicol (PEG 6000), de osmolaridade similar, foram preparadas para avaliar possíveis efeitos osmóticos dos extratos aquosos. Nos testes de germinação, as sementes de gergelim foram colocadas em placas de Petri forradas com papel de filtro com a solução a ser testada e observadas a cada $8 \mathrm{~h}$. Para os experimemtos de crescimento, sementes de gergelim foram germinadas em água e posteriormente dispostas para crescimento nos extratos. Após 5 dias, foram medidos os comprimentos da parte aérea e radicular das plântulas. Todos os experimentos foram conduzidos a $22^{\circ} \mathrm{C}, 30^{\circ} \mathrm{C}$ e $38^{\circ} \mathrm{C}$. Observou-se que os extratos de folhas não afetaram a germinabilidade, mas aumentaram o tempo médio de germinação em uma relação próxima à dose-dependente, nas três temperaturas. Quanto ao crescimento, a parte radicular foi a mais afetada pelos extratos aquosos, apresentando redução no tamanho, necroses, ausência de pêlos absorventes e formação de raízes laterais. Os efeitos dos extratos no crescimento das plântulas foram mais evidentes a $38^{\circ} \mathrm{C}$. Os experimentos conduzidos com soluções de PEG 6000 mostraram que os efeitos observados na presença dos extratos não são de natureza osmótica.
\end{abstract}

Palavras-chaves: alelopatia, crescimento, germinação, lobeira, temperatura

\begin{abstract}
Allelopathic effect of Solanum lycocarpum A. St.-Hil. leaves on the germination and growth of Sesamum indicum L. (Pedaliaceae) under different temperatures). Allelopathy should be defined as any stimulatory or inhibitory effect by one plant on another through production of chemical compounds released into the environment. Several Solanum species have shown some allelopathic property. S. lycocarpum is largely distributed on disturbed areas of the Brazilian Cerrado. In the present study the effects of aqueous extracts of $S$. lycocarpum leaves on the germination and growth of Sesanum indicum L. (sesame) were investigated. Aqueous leaf extracts at concentrations of $1 \%, 2 \%, 3 \%, 4 \%$, and $5 \%(\mathrm{w} / \mathrm{v})$ were prepared. The osmolarity of the extracts were measured and solutions of polyethylene glycol (PEG 6000) of similar osmolarity were prepared to evaluate osmotic effects of the extracts on sesame germination and growth. The experiments were carried out on petri dishes lined by two layers of filter paper plus the solutions to be tested. For the germination experiments the number of germinated seeds was checked every $8 \mathrm{~h}$. For the growth experiments sesame seeds were previously germinated in water and disposed to grow in the extracts. After five days of incubation the root and shoot length of the seedlings was measured. All the experiments were performed at $22^{\circ} \mathrm{C}, 30^{\circ} \mathrm{C}$ and $38^{\circ} \mathrm{C}$. The extracts did not affect the germinability but increased the average germination time in a dose-dependent manner at the three temperatures. The root growth was more affected by the extracts, showing tip-necrosis, absence of root hairs, and formation of secondary roots. These effects were more evident at $38^{\circ} \mathrm{C}$. Using PEG 6000 it was shown that the observed effects were not due to osmotic properties of the leaf extracts.
\end{abstract}

Key words: allelopathy, germination, plant growth, lobeira, temperature

\section{Introdução}

O Cerrado brasileiro cobre cerca de $25 \%$ do território nacional e apresenta grande diversidade de espécies e de fitofisionomias (Ribeiro \& Walter 1998).
O solo é ácido, com pouca disponibilidade de nutrientes e alta concentração de alumínio (Haridasan 2000). O Cerrado apresenta duas estações definidas, a estação chuvosa (outubro a abril) e a estação seca (maio a setembro). A ocorrência de queimadas é um evento

\footnotetext{
1 Universidade Católica de Brasília (UCB), Laboratório de Botânica, Câmpus I (Taguatinga). EPCT“'QS, Lote 1, CEP 72030-170, Águas Claras, Taguatinga, DF (sarah@ucb.br)

2 Universidade Federal Rural do Rio Grande do Sul, Pesquisador Associado Senior (UnB), C. Postal 15015, CEP 91501-970, Porto Alegre, RS, Brasil (ferreira@unb.br)

3 Departamento de Botânica, Laboratório de Termobiologia (UnB), C. Postal 04467, CEP 70910-900, Brasília, DF, Brasil

4 Autor para correspondência: fborghet@unb.br
} 
comum durante a estação seca, sendo considerado fator de grande influência na densidade arbórea do Cerrado (Coutinho 1981; 1992; Moreira 2000). Tem sido observado aumento na freqüência de queimadas, devido ao uso do fogo pelo homem no preparo de áreas para cultivo e pastagem (K.R.F. Vicentini, comunicação pessoal).

Alelopatia é definida como o efeito direto ou indireto, benéfico ou maléfico de uma planta sobre outra por intermédio da produção de compostos químicos que são liberados no meio ambiente (Rice 1984). Esses agentes alelopáticos são geralmente compostos secundários produzidos pelas plantas e liberados por lixiviação, volatilização, decomposição e exudados da raiz (Inderjit \& Dakshini 1992; 1994). A produção de compostos secundários pode ser aumentada por diversos fatores ambientais como estresse hídrico, deficiência de nutrientes e temperatura (Einhellig 1996) situações comumentes encontradas no Cerrado.

A influência da temperatura na ação dos aleloquímicos é pouco discutida na literatura. Duke et al. (1983) relataram que a $35^{\circ} \mathrm{C}$ compostos fenólicos demoravam mais tempo para agir na germinação do que a $25^{\circ} \mathrm{C}$. Em contraste, Einhellig \& Eckrich (1984) encontraram que a inibição do crescimento do sorgo e da soja por ácido ferúlico foi maior em temperaturas mais altas do que em temperaturas mais baixas. Estas observações sugerem que a ação dos aleloquímicos no desenvolvimento vegetal pode sofrer significativa influência da temperatura de incubação.

Solanum lycocarpum A. St.-Hil. (Solanaceae), conhecida popularmente como lobeira ou fruta-do-lobo, é uma espécie arbustiva de ocorrência rara na vegetação nativa (Felfili et al. 1992), mas amplamente distribuída em ambientes perturbados do Cerrado (Lombardi \& Motta Jr. 1993; Oliveira-Filho \& Oliveira 1988). Dentre os dispersores de suas sementes encontram-se o lobo-guará (Chrysocyon brachyurus Illiger), espécie de hábitos cursoriais (Lombardi \& Motta Jr. 1993), e formigas do gênero Atta (F.S. Pinto, comunicação pessoal). Sua ampla distribuição em áreas alteradas e a dispersão das sementes por agentes comuns a ambientes abertos sugerem que a lobeira é espécie importante no processo de recolonização natural de clareiras e áreas perturbadas.

Sabe-se que sementes de lobeira têm efeito alelopático sobre a germinação de Cosmos sulphureus Cav., além de apresentarem efeitos autotóxicos (Borghetti \& Pessoa 1997). Além disto, existem evidências de que o gênero Solanum apresenta efeito alelopático sobre outras plantas (Sarma 1974; Frank
1990; Smith et al. 1990). Os alcalóides presentes nas plantas deste gênero, tais como a solanina, solamargina e solasodina (Motidome et al. 1970), poderiam atuar como aleloquímicos, além de fenóis que são comumente encontrados nas folhas (Güntner et al. 1997).

Em vista destas observações, os objetivos do presente trabalho foram os de investigar efeitos alelopáticos de extratos de folhas de lobeira na germinação e no crescimento inicial de gergelim, sob três temperaturas de incubação.

\section{Material e métodos}

Extratos de folhas - Para o preparo de extratos aquosos de folhas, estas foram coletadas de indivíduos de Solanum lycocarpum A. St.-Hil. encontrados no Campus da Universidade de Brasília. Folhas recémcolhidas foram secas em estufa por 24 horas a $50^{\circ} \mathrm{C}$, maceradas, e em seguida preparou-se uma solução, dissolvendo 5 gramas do macerado em $100 \mathrm{~mL}$ de água destilada. A solução foi armazenada a $4 \pm 2^{\circ} \mathrm{C}$ por período de $24 \mathrm{~h}$, para extração aquosa (Jacobi \& Ferreira 1991). Após, a solução foi filtrada em papelfiltro Whatman \# 1 com auxílio de bomba a vácuo. A partir dessa solução foram feitas as diluições de 1, 2, 3,4 e $5 \%$. Essas soluções, contendo extrato aquoso bruto de folhas, tiveram a sua osmolaridade medida (osmômetro Wescor 5240), e soluções de PEG 6000 (VETEC) foram preparadas na mesma osmolaridade dos extratos.

Espécie utilizada nos bioensaios - Para os bioensaios de germinação e crescimento foram utilizadas sementes de gergelim (Sesamum indicum L.) da variedade "black variety", colhidas no estado de Goiás em 1998 (EMBRAPA), e armazenadas em sacos de papel a temperatura de $22 \pm 2^{\circ} \mathrm{C}$. Optou-se pelo uso do gergelim pois seus parâmetros de germinação já foram estabelecidos (Carvalho et al. 2001). As sementes foram previamente tratadas com Captan a $0,2 \%$ por 60 segundos e secas com papel-filtro.

Experimentos de germinação - Os experimentos foram conduzidos em câmaras de germinação (Marconi MA 403), sob temperaturas constantes de 22,30 e $38^{\circ} \mathrm{C}$ (variação de temperatura menor que $0,1^{\circ} \mathrm{C}$ ), na ausência de luz. Os testes foram feitos em placas de Petri de $90 \mathrm{~mm}$ diâm. e revestidas com duas folhas de papel-filtro Whatman \# 1. As placas foram autoclavadas e, em seguida, 50 sementes de gergelim previamente desinfestadas foram distribuídas nas placas. Em cada placa foram adicionados $6 \mathrm{~mL}$ da 
solução a ser testada. Para cada tratamento foram feitas 4 repetições, totalizando 200 sementes por tratamento, em cada temperatura. As placas foram dispostas aleatoriamente na câmara de germinação e o número de sementes germinadas foi verificado em intervalos de $8 \mathrm{~h}$ sob luz branca, visto tratar-se de espécie afotoblástica (Carvalho et al. 2001). O critério de germinação foi a emergência da radícula seguida do curvamento geotrópico da raiz (Labouriau 1983).

Experimentos de crescimento - Para os experimentos de crescimento foram utilizadas sementes de gergelim germinadas a $30^{\circ} \mathrm{C}$ por até $28 \mathrm{~h}$. Dez sementes germinadas foram distribuídas em placa de cultura de vidro de $150 \times 30 \mathrm{~mm}$ revestidas com duas folhas de papel-filtro e previamente autoclavadas. Em cada placa foram adicionados $10 \mathrm{~mL}$ do extrato a ser testado, e para cada tratamento foram feitas 3 repetições, totalizando 30 plântulas por tratamento, em cada temperatura. As plântulas cresceram por 5 dias nas mesmas temperaturas utilizadas nos experimentos de germinação, sob fotoperíodo de $12 \mathrm{~h}\left(400 \mu \mathrm{mol} \cdot \mathrm{m}^{-2} \cdot \mathrm{s}^{-1}\right)$. Após, as partes aérea e radicular foram medidas com auxílio de paquímetro. Aspectos morfológicos, tais como presença de raízes secundárias, pêlos radiculares e necroses também foram verificados.

Análise estatística - Foram calculados a germinabilidade e o tempo médio de germinação, segundo Labouriau (1983). Os dados, tanto de germinação quanto de crescimento, foram submetidos ao teste de ANOVA, seguido pelo teste de Tukey a 5\%. Os experimentos de germinação e crescimento foram realizados no Laboratório de Termobiologia L.G. Labouriau, da Universidade de Brasília.

\section{Resultados e discussão}

Germinação - Sementes de gergelim apresentaram alta germinabilidade (ao redor de $88 \%$ ), nas diferentes velocidades de germinação nas temperaturas utilizadas no presente trabalho. Enquanto a $22^{\circ} \mathrm{C}$ a velocidade média de germinação encontrou-se entre $0,020 \mathrm{e}$ $0,025 \mathrm{~h}^{-1}$ (tempo médio entre 50 e $40 \mathrm{~h}$, respectivamente), nas temperaturas de 30 e $38^{\circ} \mathrm{C}$ a velocidade média de germinação situou-se entre 0,040 e $0,045 \mathrm{~h}^{-1}$ (tempo médio entre 25 e 22 horas, respectivamente) (Carvalho et al. 2001). Extratos aquosos de folhas de lobeira não interferiram significativamente na germinabilidade das sementes de gergelim em nenhuma das temperaturas analisadas (Tab. 1). A ausência de efeitos alelopáticos na germinabilidade foram
Tabela 1. Efeito dos extratos aquosos de folhas de Solanum lycocarpum A. St.-Hil. e de soluções de PEG 6000 de osmolaridade similar aos extratos na germinabilidade (\%) de sementes de Sesamum indicum $\mathrm{L}$. incubadas em diferentes temperaturas.

\begin{tabular}{lccc}
\hline Tratamentos & $22^{\circ} \mathrm{C}^{* *}$ & $30^{\circ} \mathrm{C}$ & $38^{\circ} \mathrm{C}$ \\
\hline Água & $96,5 \pm 1,9 \mathrm{a}^{*}$ & $98,5 \pm 1,0 \mathrm{a}$ & $98,0 \pm 2,8 \mathrm{a}$ \\
Extrato 1\% & $97,0 \pm 2,0 \mathrm{a}$ & $99,8 \pm 0,0 \mathrm{a}$ & $98,0 \pm 1,6 \mathrm{a}$ \\
Extrato 2\% & $97,0 \pm 3,8 \mathrm{a}$ & $99,8 \pm 0,0 \mathrm{a}$ & $96,0 \pm 3,3 \mathrm{a}$ \\
Extrato 3\% & $95,5 \pm 3,0 \mathrm{a}$ & $98,0 \pm 2,8 \mathrm{a}$ & $98,5 \pm 1,9 \mathrm{a}$ \\
Extrato 4\% & $93,0 \pm 6,3 \mathrm{a}$ & $99,5 \pm 1,0 \mathrm{a}$ & $97,0 \pm 2,0 \mathrm{a}$ \\
Extrato 5\% & $94,0 \pm 3,3 \mathrm{a}$ & $98,0 \pm 2,3 \mathrm{a}$ & $95,0 \pm 3,5 \mathrm{a}$ \\
PEG 1\% & $99,0 \pm 1,2 \mathrm{a}$ & $98,0 \pm 2,3 \mathrm{a}$ & $99,0 \pm 1,2 \mathrm{a}$ \\
PEG 2\% & $99,0 \pm 1,0 \mathrm{a}$ & $98,5 \pm 1,9 \mathrm{a}$ & $99,0 \pm 1,2 \mathrm{a}$ \\
PEG 3\% & $96,5 \pm 3,0 \mathrm{a}$ & $98,0 \pm 2,8 \mathrm{a}$ & $98,0 \pm 1,6 \mathrm{a}$ \\
PEG 4\% & $96,0 \pm 2,3 \mathrm{a}$ & $98,5 \pm 1,0 \mathrm{a}$ & $98,0 \pm 1,6 \mathrm{a}$ \\
PEG 5\% & $89,5 \pm 11,4 \mathrm{a}$ & $98,5 \pm 1,9 \mathrm{a}$ & $97,5 \pm 2,5 \mathrm{a}$ \\
\hline
\end{tabular}

* Germinabilidade \pm desvio padrão (\%). ** Valores na mesma coluna seguidos pela mesma letra não diferem estatisticamente pelo teste de Tukey a $5 \%(4 \times 50$ sementes/tratamento $)$.

previamente descritos para sementes de Mimosa bimucronata (Ferreira \& Aquila 2000) e de alfafa (Medicago sativa) (Chon et al. 2000), sendo corroborados no presente trabalho.

Os tempos médios de germinação das sementes incubadas em água concordam com os valores obtidos em estudo anterior (Carvalho et al. 2001), para as três temperaturas de incubação. Em contrapartida, o tempo médio foi em geral aumentado pelos extratos aquosos, próximo a uma relação dose-resposta, nas três temperaturas de incubação (Tab. 2). Extratos acima de $3 \%$ aumentaram o tempo médio, independente da temperatura de incubação. A $30^{\circ} \mathrm{C}$, o efeito no tempo médio foi observado a partir do extrato a $2 \%$. Isto permite sugerir que ou as sementes de gergelim são mais sensíveis à ação do(s) aleloquímico(s) em temperaturas próximas dos $30^{\circ} \mathrm{C}$, ou os efeitos do(s) aleloquímico(s) são dependentes da temperatura. A potencialização dos efeitos de aleloquímicos em temperaturas mais altas foi observada com extratos de figos na germinação e crescimento de hortaliças (WeiBing et al. 2001).

Além dos efeitos no tempo médio, os extratos alteraram também o padrão de distribuição temporal da germinação, como se pode observar comparando os desvios padrões calculados para os experimentos conduzidos em água e na presença dos extratos (Tab. 2). Com poucas exceções, a presença dos extratos aumentou consideravelmente o desvio padrão da germinação, embora não fique evidente nenhuma relação dose-resposta. Isto mostra que sementes de 
gergelim apresentaram respostas heterogêneas aos extratos, algumas sementes sendo mais susceptíveis, e outras mais tolerantes ao(s) aleloquímico(s). Estes efeitos foram mais evidentes nas sementes incubadas a $22^{\circ} \mathrm{C}$.

Os resultados encontrados mostram que extratos de folhas de lobeira interferem no comportamento germinativo do gergelim, tanto atrasando o pico de germinação, com já descrito para outras espécies (Ferreira \& Áquila 2000), como aumentando a variância da germinação. Os efeitos das soluções de PEG 6000 na germinabilidade (Tab. 1) e no tempo médio de germinação (Tab. 2) não foram significativamente diferentes do controle em água, indicando que os efeitos dos extratos descritos acima não são de natureza osmótica.

Crescimento - Mesmo em água, plântulas de gergelim apresentaram diferentes graus de desenvolvimento em função da temperatura de incubação. Como seria esperado, o crescimento foi menor a $22^{\circ} \mathrm{C}$ quando comparado com temperaturas mais elevadas (Fig. 1). Entretanto, independentemente da diferença de crescimento em água, extratos aquosos de folhas de lobeira reduziram o crescimento de plântulas de gergelim, próximo a uma relação dose-dependente, nas três temperaturas de incubação (Fig. 1). A $22^{\circ} \mathrm{C}$, a inibição foi significativamente diferente do controle a partir do extrato a $2 \%$, sendo que a $30^{\circ} \mathrm{C}$ e $38^{\circ} \mathrm{C}$ o efeito mostrou-se presente em todas as concentrações

Tabela 2. Efeito de extratos aquosos de folhas de Solanum lycocarpum A. St.-Hil. e de soluções de PEG 6000 de osmolaridade similar aos extratos no tempo médio de germinação (horas) de sementes de Sesamum indicum L. incubadas em diferentes temperaturas.

\begin{tabular}{llll}
\hline Tratamentos & \multicolumn{1}{c}{$22^{\circ} \mathrm{C}^{* *}$} & \multicolumn{1}{c}{$30^{\circ} \mathrm{C}$} & \multicolumn{1}{c}{$38^{\circ} \mathrm{C}$} \\
\hline Água & $57,09 \pm 1,6 \mathrm{a}^{*}$ & $24,57 \pm 0,27 \mathrm{a}$ & $21,98 \pm 0,85 \mathrm{a}$ \\
Extrato 1\% & $62,97 \pm 3,78 \mathrm{ab}$ & $26,48 \pm 1,08 \mathrm{a}$ & $21,24 \pm 0,84 \mathrm{a}$ \\
Extrato 2\% & $64,32 \pm 2,06 \mathrm{ab}$ & $30,64 \pm 1,86 \mathrm{bc}$ & $21,82 \pm 1,15 \mathrm{a}$ \\
Extrato 3\% & $70,12 \pm 9,86 \mathrm{~b}$ & $29,96 \pm 1,37 \mathrm{~b}$ & $22,69 \pm 2,13 \mathrm{ab}$ \\
Extrato 4\% & $71,24 \pm 6,04 \mathrm{~b}$ & $32,08 \pm 0,43 \mathrm{bc}$ & $25,33 \pm 2,78 \mathrm{~b}$ \\
Extrato 5\% & $67,49 \pm 6,44 \mathrm{ab}$ & $32,72 \pm 1,33 \mathrm{c}$ & $25,67 \pm 1,09 \mathrm{~b}$ \\
PEG 1\% & $59,12 \pm 1,65 \mathrm{ab}$ & $24,61 \pm 0,21 \mathrm{a}$ & $20,85 \pm 1,03 \mathrm{a}$ \\
PEG 2\% & $59,61 \pm 2,38 \mathrm{ab}$ & $25,06 \pm 0,35 \mathrm{a}$ & $21,23 \pm 0,62 \mathrm{a}$ \\
PEG 3\% & $66,87 \pm 4,60 \mathrm{ab}$ & $25,21 \pm 0,76 \mathrm{a}$ & $21,49 \pm 0,79 \mathrm{a}$ \\
PEG 4\% & $61,75 \pm 3,15 \mathrm{ab}$ & $26,07 \pm 0,69 \mathrm{a}$ & $20,69 \pm 0,18 \mathrm{a}$ \\
PEG 5\% & $64,76 \pm 5,97 \mathrm{ab}$ & $25,50 \pm 0,78 \mathrm{a}$ & $21,58 \pm 0,29 \mathrm{a}$ \\
\hline
\end{tabular}

* Tempo médio de germinação \pm desvio padrão (em horas). ** Valores na mesma coluna seguidos pela mesma letra não diferem estatisticamente pelo teste de Tukey a 5\% (4×50 sementes/tratamento). utilizadas. A $38^{\circ} \mathrm{C}$ não houve desenvolvimento de plântulas nas concentrações 4 e $5 \%$, sendo que as sementes germinadas encontravam-se necrosadas. A porcentagem de inibição do crescimento pelos extratos, comparado ao controle (em água), mostra efeitos mais evidentes nas temperaturas de 30 e $38^{\circ} \mathrm{C}$. Esses resultados indicam que os efeitos dos extratos no crescimento das plântulas dependem da temperatura de incubação.

Dentre as alterações morfológicas observadas nas plântulas tratadas com os extratos observou-se a ausência de pêlos absorventes nas raízes. A ausência ou diminuição na quantidade de pêlos absorventes é
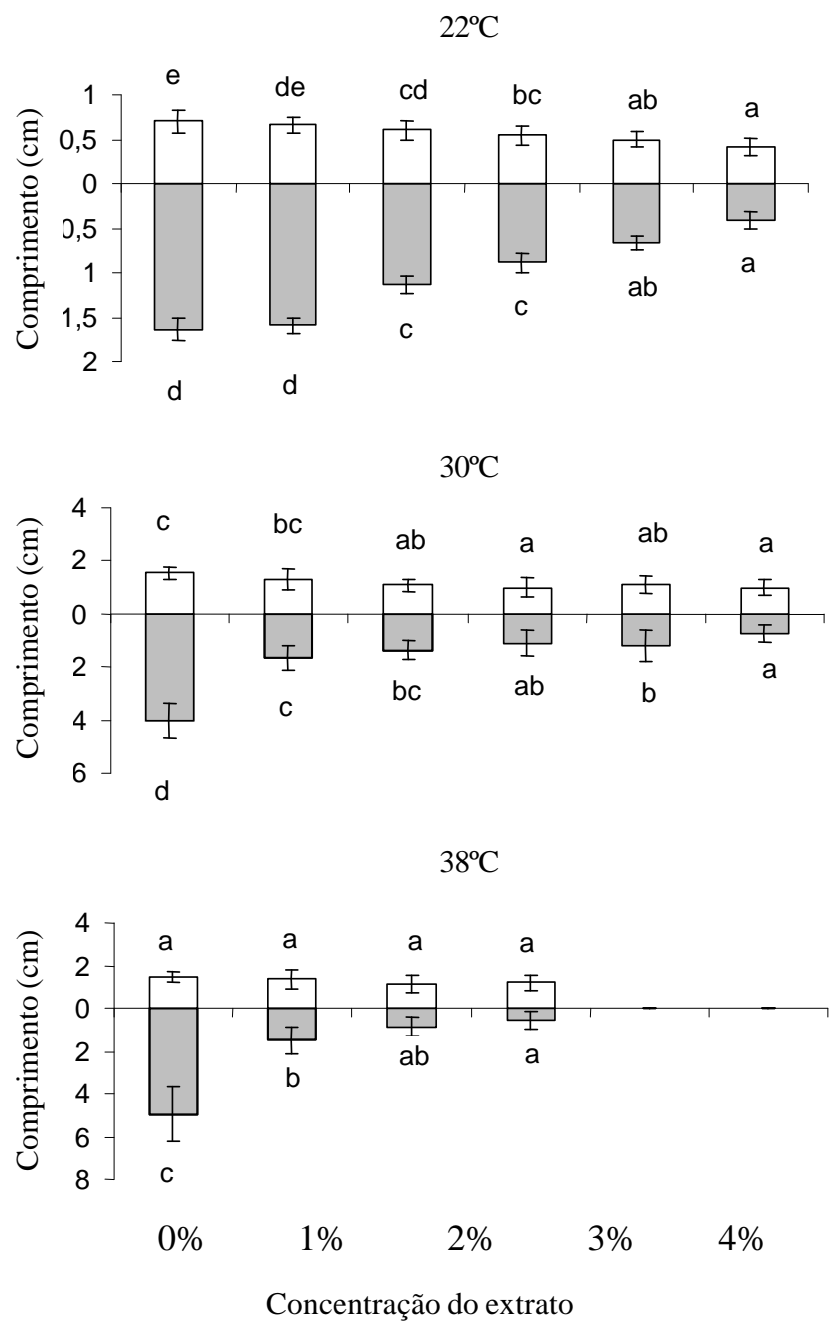

Figura 1. Tamanho médio (cm) da parte aérea $(\square)$ e radicular $(\square)$ de plântulas de Sesamum indicum L. incubadas por cinco dias em diferentes concentrações de extrato aquoso de folhas de Solanum lycocarpum A. St.-Hil. Barras verticais representam o desvio padrão. Colunas com a mesma letra não diferem estatisticamente pelo teste de Tukey a 5\%. As comparações entre as concentrações foram feitas separadamente para parte aérea e radicular (30 plântulas/tratamento). 
um parâmetro bastante sensível para experimentos de alelopatia (Miró et al. 1998). Observou-se também o aparecimento de raízes laterais nas plântulas incubadas nos extratos. A formação acentuada de raízes laterais pode estar relacionada aos distúrbios no balanço hormonal das plântulas (Dayan et al. 2000). Em diversos casos também se observou desenvolvimento incompleto da coifa e inversão no gravitropismo das raízes por efeito dos extratos. A inversão do gravitropismo foi também observada no efeito autotóxico da alfafa (Chon et al. 2000). Segundo os autores, seria uma forma de evitar o contato com os aleloquímicos.

O crescimento da parte aérea foi progressivamente reduzido pelos extratos a $22^{\circ} \mathrm{C}$ e, com menor evidência, a $30^{\circ} \mathrm{C}$; a $38^{\circ} \mathrm{C}$, entretanto, extratos entre 1 e $3 \%$ não interferiram no crescimento aéreo, enquanto a 4 e $5 \%$ não houve desenvolvimento de plântulas (Fig. 1). Morfologicamente, a parte aérea das plântulas tratadas com os extratos não se mostrou muito diferente das plântulas incubadas em água, exceto pelo alto grau de retorcimento observado. Contudo, não se pode assegurar se a redução do crescimento da parte aérea foi resultante da ação direta do(s) aleloquímico(s), ou uma consequiência da redução do crescimento da parte radicular.

De forma geral, as raízes mostraram-se mais sensíveis à ação do(s) aleloquímico(s) comparada com a parte aérea, o que já foi relatado por diversos autores (Chon et al. 2000; Ferreira \& Áquila 2000; Batish et al. 2002). Enquanto a parte radicular foi inibida cerca de $75 \%$ a $22^{\circ} \mathrm{C}$, a parte aérea chegou no máximo a $35 \%$ de inibição, na mesma concentração utilizada.

Plântulas crescidas em soluções de PEG 6000 apresentaram pequena redução do tamanho da parte aérea e, às vezes, até promoção (Fig. 2). Contudo, os sintomas apresentados pelas plântulas que cresceram na presença dos extratos não apareceram nas plântulas incubadas nas soluções de PEG 6000.

A lobeira é considerada espécie sempre verde, e suas folhas estão em constante queda e substituição. Como as folhas são relativamente grandes, elas caem ao chão e acumulam-se próximo à planta-mãe. Visto que extratos de folhas secas também apresentam propriedades alelopáticas (dados não mostrados), o acúmulo das folhas poderia eventualmente impedir o estabelecimento de outras plantas próximas à lobeira. Os efeitos desta ação química em plantas sob condições naturais precisam ser investigada.

O uso de espécies do Cerrado para estudos de alelopatia mostrou-se promissor. A identificação de propriedades alelopáticas em espécies nativas abre perspectivas de que tais efeitos possam se encontrar entre os fatores determinantes da dinâmica da vegetação e da composição florística das fitofisionomias do Cerrado.

$22^{\circ} \mathrm{C}$
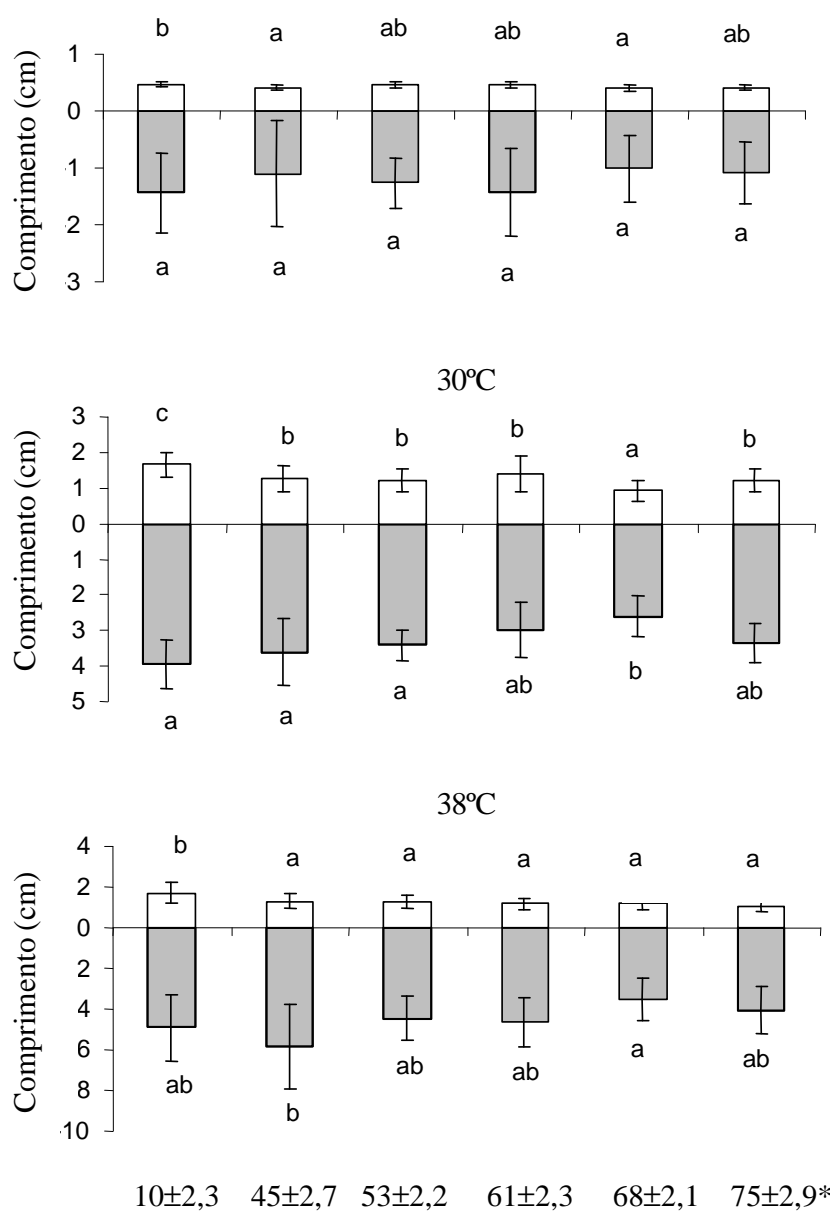

Soluções de PEG 6000

Figura 2. Tamanho médio $(\mathrm{cm})$ da parte aérea $(\square)$ e radicular $(\square)$ de plântulas de Sesamum indicum L. incubadas por cinco dias em soluções de PEG 6000 de osmolaridade similar aos extratos aquosos $(0,1,2,3,4$ e $5 \%$, respectivamente) de folhas de Solanum lycocarpum A. St.-Hil. Barras verticais representam o desvio padrão. Colunas com a mesma letra não diferem estatisticamente pelo teste de Tukey a 5\%. As comparações entre as soluções de PEG 6000 foram feitas separadamente para parte aérea e radicular (30 plântulas/tratamento). *Osmolaridade média de cinco repetições \pm desvio padrão $\left(\mathrm{mmol}^{\mathrm{kg}} \mathrm{kg}^{-1}\right)$.

\section{Agradecimentos}

Os autores agradecem ao Dr. Augusto C. Franco (UnB), pelo empréstimo do osmômetro; ao CNPq, pelo financiamento do projeto e bolsas; à direção do Parque Nacional de Brasília, IBAMA, pelo apoio; à CAPES, pela bolsa de Mestrado da primeira Autora. 


\section{Referências bibliográficas}

Batish, D.R.; Singh, H.P.; Kohli, R.K.; Saxena, D.B. \& Kaur, S. 2002. Allelopathic effects of parthenin against two weedy species, Avena fatua and Bidens pilosa. Environmental and Experimental Botany 47: 149-155.

Borghetti, F. \& Pessoa, D.M.A. 1997. Autotoxicidade e alelopatia em sementes de Solanum lycocarpum St. Hil. (Solanaceae). Pp. 54-58. In: L.L. Leite \& C.H. Saito (orgs.). Contribuição ao conhecimento ecológico do cerrado. Editora da UnB, Brasília, DF.

Carvalho, P.G.B.; Borghetti, F.; Buckeridge, M.S.; Morhy, L. \& Ferreira-Filho, E.X.F. 2001. Temperature-dependent germination and endo-b-mannase activity in sesame seeds. Revista Brasileira de Fisiologia Vegetal 13: 139-148.

Chon, S.U.; Coutts, J.H. \& Nelson, C.J. 2000. Effects of light, growth media, and seedling orientation on biossays of alfalfa autotoxicity. Agronomy Journal 92: 715-720.

Coutinho, L.M. 1981. Aspectos ecológicos do fogo no cerrado - Nota sobre ocorrência e a datação de carvões vegetais encontrados no interior de solo sob cerrado. Revista Brasileira de Botânica 4: 115-117.

Coutinho, L.M. 1992. O cerrado e a ecologia do fogo. Ciência Hoje Volume especial: 130-138.

Dayan, F.E.; Romagni, J.G. \& Duke, S.O. 2000. Investigating the mode of action of natural phytotoxins. Journal of Chemical Ecology 26: 2079-2094.

Duke, S.O.; Williams, R.D. \& Markahart, A.H.I. 1983. Interaction of moisture stress and three phenolic compounds on lettuce seed germination. Annals of Botany 52: 923-926.

Einhellig, F.A. 1996. Interaction involving allelopathy in crop systems. Agronomy Journal 88: 886-893.

Einhellig, F.A. \& Eckrich, P. 1984. Interactions of temperature and ferulic acid stress on grain sorghum and soybeans. Journal of Chemical Ecology 10: 161-170.

Felfili, J.M.; Silva-Jr, M.C.; Rezende, A.V.; Machado, J.W.B.; Walter, B.M.T.; Silva P. \& Hay, J.D. 1992. Análise comparativa da florística e fitossociologia da vegetação arbórea do cerrado sensu stricto na Chapada Pratinha, DF, Brasil. Acta Botanica Brasilica 6: 27-46.

Ferreira, A.G. \& Áquila, M.E.A. 2000. Alelopatia: uma área emergente da ecofisiologia. Revista Brasileira de Fisiologia Vegetal 12(edição especial): 175-204.

Frank, J.R. 1990. Influence of horsenettle (Solanum carolinense) on snapbean (Phaseolus vulgaris). Weed Science 38: 220-223.

Güntner, C.; Gonzales, A.; Reis, R.; Gonzalez, G.; Vazquez, A.; Ferreira, A. \& Moyna, P. 1997. Effect of Solanum glycoalkaloids on potato aphid, Macrosiphum euphorbiae. Journal of Chemical Ecology 23: 1651-1659.
Haridasan, M. 2000. Nutrição mineral de plantas nativas do cerrado. Revista Brasileira de Fisiologia Vegetal 12: 54-64.

Inderjit \& Dakshini, K.M.M. 1992. Interference potential o Pluchea lanceolata (Asteraceae): growth and physiological responses of asparagus bean, Vigna unguiculata var. sesquipedalis. American Journal of Botany 79: 977-981.

Inderjit \& Dakshini, K.M.M. 1994. Allelopathic effect of Pluchea lanceolata (Asteraceae) on characteristics of four soils and tomato and mustard growth. American Journal of Botany 81: 799-804.

Jacobi, U.S. \& Ferreira, A.G. 1991. Efeitos alelopáticos de Mimosa bimucronata (DC)OK sobre espécies cultivadas. Pesquisa Agropecuária Brasileira 26: 935-943.

Labouriau, L.G. 1983. A germinação das sementes. Washington, OEA.

Lombardi, J.A. \& Motta Jr., J.C. 1993. Seed dispersal of Solanum lycocarpum St. Hil. (Solanaceae) by the maned wolf, Chrysocyon brachyurus Illiger (Mammalia, Canidae). Ciência e Cultura 45: 126-127.

Miró, C.P.; Ferreira, A.G. \& Áquila, M.E. 1998. Alelopatia de frutos de erva-mate (Ilex paraguariensis) no desenvolvimento do milho. Pesquisa Agropecuária Brasileira 33: 1261-1270.

Moreira, A.G. 2000. Effects of fire protection on savanna structure in Central Brazil. Journal of Biogeography 27: 1021-1029.

Motidome, M.; Leekning, M.E. \& Gottlieb, O.R. 1970. A química das Solanaceas brasileiras I. A presença de solamargina e de solasodina no juá e na lobeira. Anais da Academia Brasileira de Ciências 42: 375-376.

Oliveira-Filho, A.T. \& Oliveira, L.C.A. 1988. Biologia floral de uma população de Solanum lycocarpum St. Hil. (Solanaceae) em Lavras. Revista Brasileira de Botânica 11: 23-32.

Ribeiro, J.F. \& Walter, B.M.T. 1998. Fitofisionomias do bioma Cerrado. Pp.89-166. In: S.M. Sano \& S.P. Almeida. Cerrado: ambiente e flora. Planaltina, DF: Embrapa, CPAC

Rice, E.L. 1984. Allelopathy. $2^{\text {nd }}$ ed. Academic Press, New York.

Smith, B.S.; Pawlak, J.A.; Murray, D.S.; Verhaden, L.M. \& Green, J.D. 1990. Interference from established stands of silverleaf nightshade (Solanum elaeagnifolium) on cotton (Gossypium hirsutum) lint yield. Weed Science 38: $129-133$.

WeiBing, J.; Kai, M.; WB, J. \& K, M. 2001. Effects of extracts from fig (Ficus carica) organs on the germination of seeds and the growth of seedling of several vegetable and legume crops. Journal of Fruit Science 18: 39-42. 